\title{
Increasing the Role of Practice Networks in Medical Research
}

\author{
Philip D. Sloane, MD, MPH, Rowena J. Dolor, MD, MHS, and \\ Jacquie Halladay, MD, MPH
}

An often-unrecognized consequence of the rise of generalist departments and divisions in US medical schools has been an improved sense of affiliation between physicians in community practice and schools of medicine. Generalists-in-training now rub shoulders with generalist researchers; continuing education for primary care providers is frequently conducted by primary care faculty; and generalist faculty increasingly contribute to the practice-relevant research literature. All these things have made today's family physicians, general pediatricians, and general internists feel a greater kinship with schools of medicine and, consequently, a greater willingness to participate in research.

The most overt expression of this increased willingness to participate in research has been the remarkable rise in practice-based research networks (PBRNs). Beginning in the 1980s and growing slowly in the 1990s, the PBRN movement has accelerated markedly during the past decade, thanks in large measure to encouragement and support from the US Agency for Healthcare Research and Quality (AHRQ), the Robert Wood Johnson Foundation's Prescription for Health Program, and

Submitted 5 May 2009; revised 5 May 2009; accepted 5 May 2009.

From the North Carolina Network Consortium (PDS, JH, RJD); the Department of Family Medicine and the Cecil G. Sheps Center for Health Services Research, University of North Carolina at Chapel Hill (PDS, JH); and the Duke Clinical Research Institute and Department of Medicine, Duke University, Durham, North Carolina (RJD).

Funding: none.

Conflict of interest: none declared.

Corresponding author: Philip D. Sloane, MD, MPH, Department of Family Medicine, University of North Carolina, Chapel Hill, NC 27599-7595 (E-mail: psloane@med.unc. edu).

\section{See Related Articles on Pages 446 and 453.}

the emphasis being placed on community engagement by the National Institutes of Health's Clinical and Translational Science Award program. Still, the true heroes in the growth of PBRNs are the primary care clinicians and staff who have been willing to engage and participate in research, despite the increasingly burdensome nature of everyday practice.

\section{The Current State of PBRN Research}

Research in PBRNs is burgeoning, as is evidenced by this-the fourth annual practice-based research theme issue. PBRN research is no doubt published in many venues, some of which may make identification of a given study as "PBRN research" difficult. However, one window on the current state of PBRN research is the bibliography maintained by the AHRQ on its PBRN website. ${ }^{1}$

To better understand the current status of PBRN research we evaluated all the articles posted on the AHRQ bibliography as having been published in 2007 and 2008. After eliminating redundant entries, unpublished presentations, studies that were not actually conducted by PBRNs, and the 30 articles that were about PBRN development and research methods, we were left with 52 publications of PBRN-conducted research. This provided us with a snapshot of the types of research currently being conducted in PBRNs.

Table 1 summarizes the content and methods of these 52 PBRN studies. About half had their primary focus on the providers and practices, such as describing how medical tasks are performed and what changes could be made to improve the status quo. For the other studies, PBRNs provided researchers with access to patient populations for studies of patient behavior and/or of conditions that are encountered in the primary care setting. Methodologically, descriptive approaches predominated, primarily using survey methods but with a 
Table 1. Content and Methods of 52 Practice-Based Research Network Studies Published in 2007 and 2008

\begin{tabular}{lc}
\hline & Studies (n) \\
\hline Study Content & \\
Practice/provider as unit of observation & 30 \\
Provider behavior and attitudes & 11 \\
Practice improvement/intervention trials & 19 \\
Patient as unit of observation & 22 \\
Patient behavior and attitudes & 11 \\
$\quad$ Epidemiology of common medical problems & 10 \\
Study Methods & 30 \\
Survey/other epidemiological & 4 \\
Qualitative & 1 \\
Case control & 5 \\
Quasi-experimental & 12 \\
Randomized trial & \\
\hline
\end{tabular}

few qualitative studies and one case-control design. The remaining one third of studies involved interventions, including 5 quasi-experimental studies and 12 randomized trials.

These results indicate that PBRNs are providing researchers with a window to both the providers and the patients in community medical practices, and that a wide variety of research designs, including sophisticated randomized trials, are being conducted in these settings. Such a range of topics and methods is mirrored in the selections in this special issue.

\section{What PBRNs Can and Cannot Do}

For PBRNs to grow into a mature, productive, and sustainable research enterprise, the PBRN movement must evolve. Currently, more than 100 PBRNs exist but many are cobbled together more with enthusiasm and promise rather than a developing body of research and research expertise, and few have significant ongoing infrastructural support. Furthermore, the current spate of articles about conducting research in PBRNs, including those by Yawn et $\mathrm{al}^{2}{ }^{2}$ and Williams et $\mathrm{al},{ }^{3}$ in this journal, provides testimony to the need for maturation of PBRNs as a research entity.

One aspect of this maturation must be a clearer understanding of what research can be done best in community medical practices. From a disease perspective, PBRNs are ideal places in which to study both the epidemiology and management of common medical problems, including virtually all the leading causes of death and disability. Furthermore, primary care practices see high proportions of patients with risk factors for chronic disease, such as obesity, hypertension, smoking, poor nutrition, and physical inactivity, and therefore are excellent sites in which to study interventions related to disease prevention. ${ }^{4}$ From a health services perspective, PBRNs are ideal settings to enroll patients for studies of health behavior, to engage clinicians for studies of medical care provision and practice change, and to test innovations. Particularly critical to the improvement of health at a national level is the potential role of PBRNs in understanding the differences between what experts recommend and what practitioners actually do, and in testing interventions that will help bridge the chasm between efficacy and effectiveness. ${ }^{5,6}$ Finally, community practice is the only setting in which to evaluate and understand the costs and benefits of process changes such as implementing electronic health technologies and participating in the wide array of both voluntary and involuntary quality improvement initiatives, ${ }^{7}$ thereby informing policy makers with data and not just with affect or anecdote.

Equally important is an appreciation of what PBRNs are less well suited to do. Rare disease research is probably better conducted in referral centers where such conditions are concentrated. Similarly, basic research, phase 1 and 2 clinical trials, and bench-to-bedside translational research are more efficiently conducted in major medical centers, where scientists, methodologists, and scientific equipment are more readily available. From a practical perspective, the clinical focus and general frenetic pace of primary care practices mitigates against their common use for studies involving complex protocols, multiple subspecialists, and long data collection efforts. In addition, although there is less selection bias in primary care than in tertiary care, one must nevertheless be cautious about making statistical inferences about populations in general, or even about practices in general, from studies conducted in PBRNs. ${ }^{4,8}$

Randomized controlled trials provide a special opportunity and challenge for PBRNs. Although we believe that the role of PBRNs in fielding clinical trials should be generally expanded, the fielding of such studies must be done with sensitivity to the practice setting and a willingness to modify methods to accommodate patient preferences. Intervention trials, including drug studies, are, for example, better off using usual care controls rather than 
placebo controls. To allow patients to express preferences and still be enrolled in a study, innovative approaches such as Zelen's randomized consent design or Brewin and Bradley's partially randomized patient-centered design should be considered. ${ }^{9}$ Finally, to make clinical trials widely feasible, researchers must ensure that personnel are available on site who have the skill level and the time necessary to adequately inform patients during the consenting process and to follow detailed protocols.

\section{Toward Greater PBRN Involvement in Research: Features of the Mature PBRN}

The vast access to large numbers of patients, providers, and primary care environments offered by PBRNs can be an immense asset for stakeholders striving to answer important clinical questions and evaluate the impact of innovations in health care reform and policy. Further growth and maturation of the PBRN movement is needed to fully realize this potential, however, because the mature PBRN must have several features that support high quality community-based research.

The mature PBRN should serve a clearly defined geographic area, one in which multiple practices of varying types and sizes are available for research and representation of ethnic and racial minorities is assured. Electronic databases must be available to compile and store information profiling these practices and their respective patient populations, providers, and research interests.

The mature PBRN should have ready access to a wide range of relevant research expertise. A core set of scientists and staff with knowledge and experience in sampling, instrument design, biostatistics, and proposal development must be available. Specialized skills are also necessary for practice identification and recruitment, remote data collection and monitoring, and working with institutional review boards to protect human subjects while minimizing unnecessary demands on clinicians and patients. Expertise in cost estimation and budget development are also vital.

The mature PBRN will generally carry out data collection without creating a significant burden on providers or practice staff; the pace of primary care practice requires that research not compete with patient care. One approach is to have a few "research practices," each of which would have a part-time or full-time nurse coordinator, a model that currently exists in a small number of practices for whom participation in drug trials is a significant activity. A preferable alternative, however, would be to have coordinators shared by multiple practices, thereby making research participation accessible to many more settings, providers, and patients. In this respect, a particularly useful model is one that uses a practice facilitator, a research assistant who is assigned to several practices and not only carries out data collection but also to serves as a resource for activities such as quality improvement and electronic system implementation. ${ }^{10}$ Another model uses a mobile research coordinator employed by the PBRN who travels between practices and solely focuses on recruitment and data collection so the practice staff can concentrate on clinical care.

In addition, the maturation of PBRNs will require that successful, sustainable balances be struck between the need to involve practices in research planning and the need to not demand too much of them. The community-based participatory research model, ${ }^{11}$ in which those being studied are involved at every step along the way, offers a useful starting point, although actual implementation will vary by practice and project. Depending on the researcher question and study population, researchers using a community-based participatory research approach may need to partner not only with physicians but also with other providers, office staff, and/or patients. ${ }^{12}$

Perhaps the final obstacle that must be overcome in a PBRN's maturation is that of assuring financial support for its research infrastructure. To some extent, this infrastructural support can be built into research grants; however, some guaranteed funding is needed as well. In Britain this has been addressed by providing National Health Service support to a number of regional practice networks. In the United States, a more feasible alternative would be to have PBRN support be an essential part of $\mathrm{Na}$ tional Institutes of Health funding through the Clinical and Translational Science Award program, and indeed this is occurring in some funded institutions. ${ }^{6,13}$

In conclusion, we applaud the growth of PBRNs but acknowledge that further maturation is needed for them to fulfill their promise. However, much progress has been made during the past decade, and 
we look forward to an increasingly important role for PBRNs in future clinical and translational research.

\section{References}

1. Agency for Healthcare Research and Quality. Practice-based research networks. Available at: http:// pbrn.ahrq.gov/portal/server.pt. Accessed May 12, 2009.

2. Yawn BP, Graham DG, Bertram SL, et al. Practicebased research network studies and institutional review boards: two new issues. J Am Board Fam Med 2009;22:453-60.

3. Williams RL, McPherson L, Kong A, Skipper B, Weller N, on behalf of PRIME Net clinicians. Internet-based research training in a practice-based research network consortium: a report from the Primary Care Multiethnic Network (PRIME Net). J Am Board Fam Med 2009;22:446-52.

4. Sloane PD, Callahan L, Kahwati L, Mitchell CM. Development of a Practice-Based Patient Cohort for Primary Care Research. Family Med 2006;38:50-7.

5. Sung NS, Crowley WF, Genel M, et al. Central challenges facing the national clinical research enterprise. JAMA 2003;289:1278-87.

6. Westfall JM, Mold J, Fagnan L. Practice-based re- search-"blue highways" on the NIH roadmap. JAMA 2007;297:403-6.

7. Linder JA, Ma J, Bates DW, Middleton B, Stafford RS. Electronic health record use and the quality of ambulatory care in the United States. Arch Intern Med 2007;167:1400-5.

8. Fagnan LJ, Morris C, Shipman SA, et al. Characterizing a practice-based research network: Oregon rural practice-based research network (ORPRN) survey tools. J Am Board Fam Med 2007;20:204-19.

9. Bradley C. Designing medical and educational intervention studies. Diabetes Care 1993;15:509-18.

10. Nagykaldi Z, Mold JW, Robinson A, Niebauer L, Ford A. Practice facilitators and practice-based research networks. J Am Board Fam Med 2006;19: 506-10.

11. Israel BA, Eng E, Schulz AJ, Parker EA. Methods in community-based participatory research for health. Hoboken (NJ): John Wiley \& Sons; 2005.

12. Westfall JM, VanVorst RF, Main DS, Herbert C. Community-based participatory research in practice-based research networks. Ann Fam Med 2006; 4:8-14.

13. Schwenk TL, Green LA. The Michigan clinical research collaboratory: following the NIH roadmap to the community. Ann Fam Med 2006;4(suppl 1):S49_ S54. 